\title{
Less Is More: Enhancement of Second-Harmonic Generation from Metasurfaces by Reduced Nanoparticle Density
}

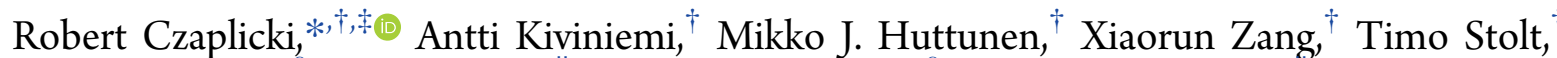

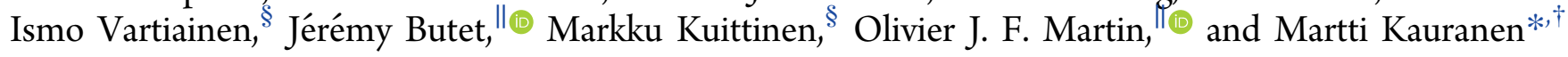

${ }^{\dagger}$ Laboratory of Photonics, Tampere University of Technology, P.O. Box 692, FI-33101 Tampere, Finland

${ }^{\ddagger}$ Institute of Physics, Faculty of Physics, Astronomy, and Informatics, Nicolaus Copernicus University, Grudziadzka 5/7, 87-100

Torun, Poland

${ }^{\S}$ Institute of Photonics, University of Eastern Finland, P.O. Box 111, FI-80101 Joensuu, Finland

"Nanophotonics and Metrology Laboratory (NAM), Swiss Federal Institute of Technology, Lausanne (EPFL), 1015 Lausanne, Switzerland

\section{Supporting Information}

ABSTRACT: We investigate optical second-harmonic generation (SHG) from metasurfaces where noncentrosymmetric Vshaped gold nanoparticles are ordered into regular array configurations. In contrast to expectations, a substantial enhancement of the SHG signal is observed when the number density of the particles in the array is reduced. More specifically, by halving the number density, we obtain over 5-fold enhancement in SHG intensity. This striking result is attributed to favorable interparticle interactions mediated by the lattice, where surfacelattice resonances lead to spectral narrowing of the plasmon resonances. Importantly, however, the results cannot be explained

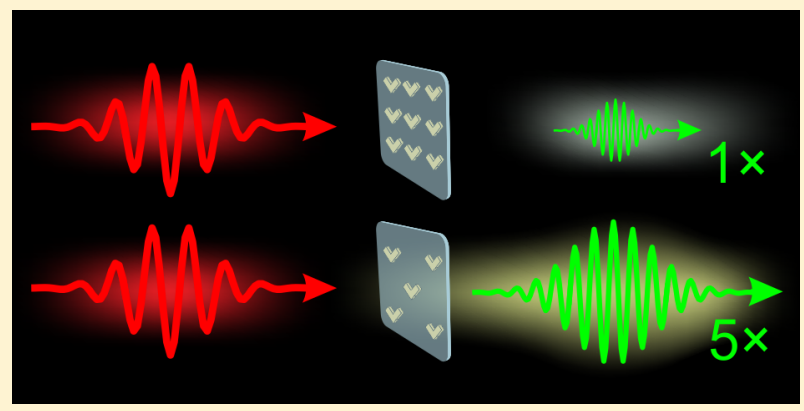
by the improved quality of the plasmon resonance alone. Instead, the lattice interactions also lead to further enhancement of the local fields at the particles. The experimental observations agree very well with results obtained from numerical simulations including lattice interactions.

KEYWORDS: Metal nanoparticles, nonlinear optics, second-harmonic generation, plasmonic resonances, surface-lattice resonances, interparticle interactions

S urface plasmons are collective oscillations of conduction electrons, which determine the optical responses of metals. When surface plasmons are excited in metal nanoparticles, they become localized and can exhibit resonant behavior. These resonances, known as localized surface plasmon resonances (LSPRs), can strongly enhance the local optical fields near the particles. ${ }^{1}$ Because the local fields rule the light-matter interactions at the nanoscale, their enhancement has facilitated the development of numerous applications based on metal nanoparticles, ranging from optical antennas, perfect lenses, light trapping structures in solar cells, surface-enhanced spectroscopies to sensing. ${ }^{2-9}$ Importantly, the strengths of light-matter interactions can be further boosted by arranging nanoparticles into periodic lattices, also known as metasurfaces. In such systems, the nanoparticles are coupled to each other either through near-field interactions ${ }^{10}$ or through their scattered fields. In the latter case, the samples can exhibit collective responses known as surface-lattice resonances (SLRs) with modified spectral features. ${ }^{11-16}$

Nonlinear optics is crucial in many photonic applications ranging from entangled-photon generation ${ }^{17}$ to frequency combs. ${ }^{18}$ However, the intrinsic material nonlinearities are often very weak, making it challenging to realize efficient nonlinear photonic devices with small footprints and reduced power requirements. The possibility to enhance local fields by utilizing LSPRs and SLRs of metasurfaces can thus be especially important for nonlinear optics, where the lightmatter interactions scale with higher powers of the fields. For example, second-harmonic generation ( $\mathrm{SHG}$ ) is a nonlinear optical process where two input fields oscillating at a fundamental frequency are combined into an output field oscillating at the doubled frequency. The efficiency of the SHG process scales with the fourth (second) power of the input field amplitude (intensity) and is thus very sensitive to changes in the local fields. ${ }^{19}$

The nonlinear properties of metal nanoparticles have been investigated for individual particles ${ }^{20-25}$ and metasurfaces. $^{10,26-35}$ In the latter case, one can envision two strategies to enhance the overall nonlinear response. By treating each nanoparticle as an elementary source of coherent nonlinear

Received: August 21, 2018

Revised: November 2, 2018

Published: November 13, 2018 
radiation, one expects the nonlinear response to scale with the square of the particle number density $N$. On the other hand, by operating close to the plasmon resonance of the nanoparticles, the response is expected to depend also on the quality of the resonance. $^{35,36}$ An attempt has been made to enhance the overall response by increasing the number of nanoparticles on a metasurface. Unfortunately, however, this compromised the quality of the resonance through interparticle interactions, ${ }^{10}$ limiting the achievable nonlinearity through this approach.

It therefore appears that any further enhancements in nonlinear responses need to be based on improving the quality of the resonances, that is, on designing metasurfaces with very narrow line widths. However, the possibilities to reduce the line widths associated with LSPRs to a greater degree are seemingly limited, because metals are intrinsically lossy materials. ${ }^{2,3,37}$ Despite this common belief, this is not a fundamental limitation because utilization of SLRs can result in spectral features with remarkably narrow line widths. ${ }^{12,38,39}$ Despite of these opportunities, only a few reports exist where SLRs have been used to enhance nonlinear responses of metasurfaces. ${ }^{40-42}$ In particular, recent theoretical predictions based on nonlinear discrete-dipole approximation (DDA) suggest that the nonlinear responses of metasurfaces can be enhanced by several orders of magnitude in the presence of SLRs. $^{39,42}$ However, in the two experiments so far $^{40,41}$ the measurements were performed as a function of the angle of incidence, hence, the effects of SLRs are mixed with the SHG radiation pattern from individual particles. ${ }^{43}$

In this Letter, we provide a striking example how SLRs can boost nonlinear responses. We demonstrate that a decrease in the particle number density of a metasurface can significantly enhance its nonlinear response. In particular, we show that the SHG efficiency of a metasurface is enhanced over 5-fold by halving the number of nanoparticles of the metasurface compared to a reference array. Although both arrays consist of identical V-shaped gold nanoparticles and exhibit similar linear responses, the arrangement of the elements in the modified metasurface results in favorable interparticle interactions and in the enhancement of SHG. The experimental results are validated by finding a good agreement with numerical simulations based on nonlinear DDA approach ${ }^{42}$ and further confirmed by simulations based on surface integral equations $^{34}$ and finite element method. ${ }^{44}$

We fabricated two-dimensional arrays of gold nanoparticles by using electron-beam lithography and lift-off techniques. The $20 \mathrm{~nm}$ thick nanoparticles were separated from the fused silica $\left(\mathrm{SiO}_{2}\right)$ substrate by a $3 \mathrm{~nm}$ thick adhesion layer of chromium and were covered from the top by a $20 \mathrm{~nm}$ thick protective layer of $\mathrm{SiO}_{2}$. Note that such a thin dielectric layer on top of the nanoparticle array also facilitates coupling of LSPRs to diffractive orders of the array, thus favoring formation of SLRs. ${ }^{14}$ The nanoparticles were designed to be V-shaped nanoantennas consisting of two equal arms (with length $l=$ $275 \mathrm{~nm}$, and width $w=100 \mathrm{~nm}$ ) oriented to each other to form an angle of $90^{\circ}$ (see Figure 1a). This shape was chosen, as it generates electric-dipole allowed SHG emission also at normal incidence. However, other shapes, such as triangles or split-ring resonators, could also be used. ${ }^{10,42}$ The particles were arranged into square lattices with periods $p_{x}=p_{y}=500 \mathrm{~nm}$. In the reference sample (V1), all the lattice points of the array were filled with particles, resulting in a square unit cell with an area of $500 \times 500 \mathrm{~nm}^{2}$, occupied thus by one particle (see dotted-line square in Figure 1a). The second sample (V2) was
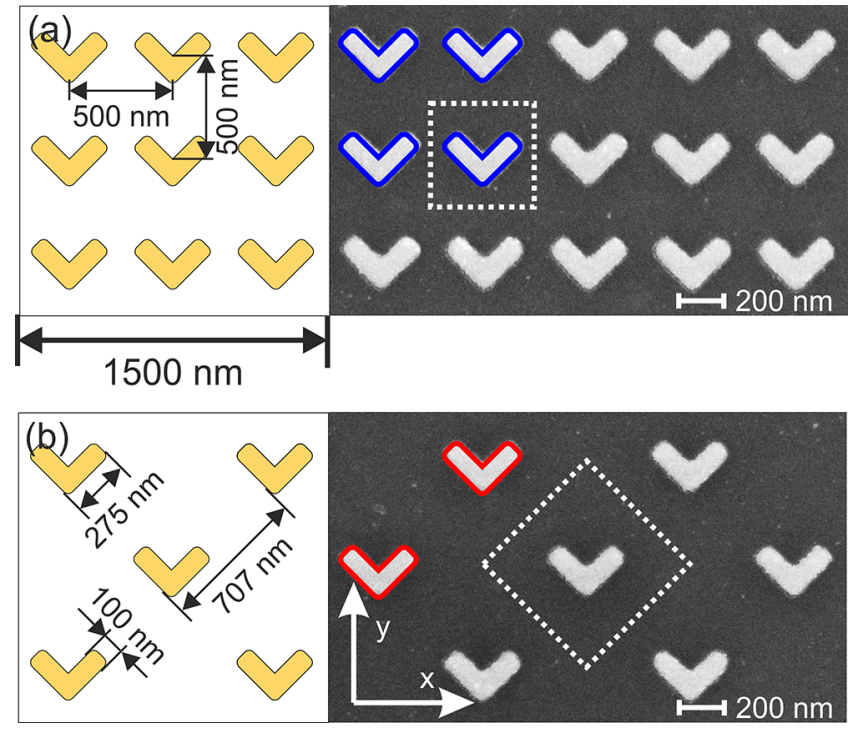

Figure 1. Design and scanning electron microscopy images of the reference sample V1 (a) and the modified sample V2 (b). The designs show a portion of the sample area with $3 \times 3$ lattice points of the square array with $500 \mathrm{~nm}$ period and the SEM images area with $5 \times 3$ lattice points. Blue (a) and red (b) outlines of nanoparticles highlight the number of particles in a $1 \times 1 \mu \mathrm{m}^{2}$ area. Dotted-line squares indicate the unit cells of the arrays. The coordinate system used for all samples is shown as an inset in (b). The dimensions of the nanoparticles and the distances between them are shown in the designs.

designed by removing the particles from every second lattice point (both in $x$ - and $y$-directions), resulting in halved particle number density $N$ compared to the reference sample V1, which was thus expected to result in 4-fold decrease in the detected SHG signal. In other words, the remaining particles formed a new lattice rotated by $45^{\circ}$ from the original one with new periods of $\sim 707 \mathrm{~nm}$ in the directions $-x+y$ and $x+y$ (see dotted-line square in Figure $1 \mathrm{~b}$ ). The V-shaped particles support LSPRs excited most efficiently by two orthogonally oriented input polarizations along the $x$ - and $y$-directions, ${ }^{45}$ as determined by the symmetry of the particles.

We first characterized the samples by linear extinction measurements. These measurements were performed by illuminating the samples with a collimated beam (diameter $\sim 1 \mathrm{~mm}$ ) from a halogen lamp and using linearly polarized light along $x$ - and $y$-directions. The spectral position of the $y$ polarized resonance is in the spectral window of 1000-1300 nm (Figure 2a), which matches closely to the fundamental wavelength used in the SHG measurements. These results also agree very well with the numerical simulations (see Figure S1 in Supporting Information). Note that the $y$-polarized spectrum of the modified sample V2 is reduced in strength, due to reduced particle density $N$. However, the reduction in extinction is less than the reduction in number density. In addition, the resonance of the modified sample V2 is redshifted and its line width is narrower than that of the reference V1. All these effects provide evidence of the excitation of SLRs in sample V2. In particular, the arrow in the Figure 2a shows the position of the Rayleigh anomaly (RA) for sample V2 that is related to the diffraction orders $( \pm 1,0),(0, \pm 1)$ of the array. Other possible RAs, outside the spectral range of our interest $(1000-1300 \mathrm{~nm})$, are not shown. The described RA is responsible for the formation of SLR in the case of sample V2 


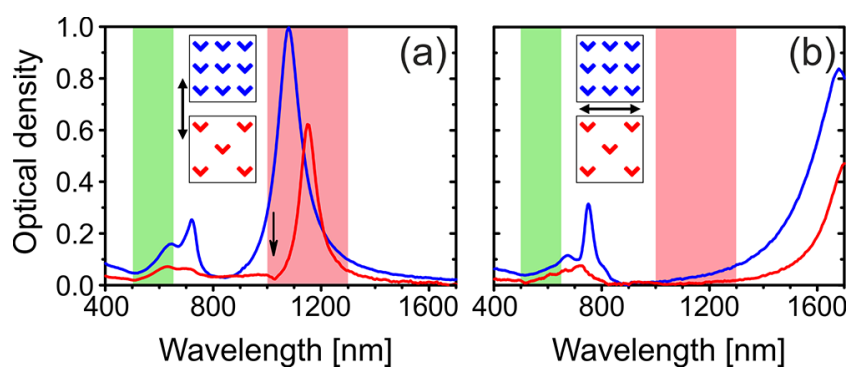

Figure 2. Extinction spectra for V1 (blue) and V2 (red) samples for (a) $y$ - and (b) $x$-polarized excitation (the direction of polarization is indicated by the double-side arrows next to the sample designs). The colored red (green) areas highlight the fundamental (SHG) wavelength range.

and is also the origin of the redshift of the $y$-polarized resonance with respect to the resonance of sample V1. ${ }^{40}$

The nonlinear properties of the samples were studied by measuring their second-harmonic ( $\mathrm{SH}$ ) responses. A normally incident fundamental beam was provided by a pulsed laser (Chameleon Vision II, Ti:sapphire, $80 \mathrm{MHz}$, pump wavelength, $770 \mathrm{~nm}$ ) combined with optical parametric oscillator (OPO; Chameleon Compact, 1000-1300 nm, pulse length, $200 \mathrm{fs}$ ) and SH light was detected in transmission (Figure 3).

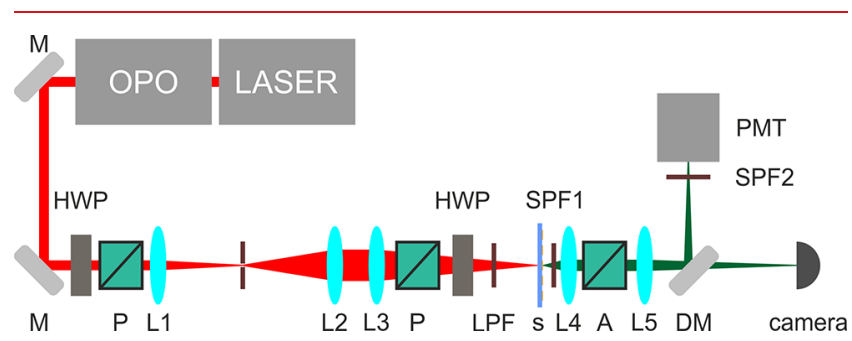

Figure 3. Schematic representation of the spectral SHG setup. M, mirrors; HWP, motorized half-wave plates; P, polarizers; L, lenses $(\mathrm{L} 1, f=30 \mathrm{~mm} ; \mathrm{L} 2, f=150 \mathrm{~mm} ; \mathrm{L} 3, f=150 \mathrm{~mm} ; \mathrm{L} 4, f=16 \mathrm{~mm} ; \mathrm{L} 5$, $f=150 \mathrm{~mm}$; L1, L2, L3, and L5, achromatic); LPF, long-pass filter $900 \mathrm{~nm}$; SPF1, short-pass filter $700 \mathrm{~nm}$; A, film polarizer (analyzer); DM, dichroic mirror; SPF2, short-pass filter $900 \mathrm{~nm}$; PMT, photomultiplier tube (PicoQuant PMA-C 192-M).

The power of the fundamental beam from the OPO was controlled by a motorized achromatic half-wave plate (HWP) and a polarizer and was set to $8 \mathrm{~mW}$. This power level was well below the estimated damage threshold level of $\sim 40 \mathrm{~mW}$ for the studied samples, and was verified to result in stable SHG signals with the expected quadratic dependence on input power (see Section 6 in Supporting Information). A set of lenses and an aperture (diameter $25 \mu \mathrm{m}$ ) were used to clean and expand the beam before entering the polarization-control part of the setup. We used an achromatic lens of $150 \mathrm{~mm}$ focal length to weakly focus the beam on the sample arrays. This ensured that the size of the excitation beam was relatively small (diameter of the beam waist was $\sim 100 \mu \mathrm{m}$ ) while still being close to a plane wave. On the basis of the above parameters, we estimate to have $\sim 12 \mathrm{MW} / \mathrm{cm}^{2}$ peak intensities at the sample plane. A high-quality polarizer and an achromatic HWP were used to control the input polarization, whereas a film polarizer after the sample was used to select the polarization of the emitted SHG light. In the experiments, the input and the detected SHG beams were both set to be $y$-polarized. A 900 $\mathrm{nm}$ long-pass (700 $\mathrm{nm}$ short-pass) filter was used to pass (block) the fundamental beam. A lens of focal length of $16 \mathrm{~mm}$ was used after the sample to efficiently collect the generated $\mathrm{SH}$ signal. Another achromatic lens of $150 \mathrm{~mm}$ focal length focused the SHG signal on the active area of a photomultiplier tube (PMT) module after being reflected by a dichroic mirror and passing through another short-pass filter $(900 \mathrm{~nm})$. In order to convert the detected SHG signals to absolute powers, a power calibration was performed on the PMT using a radiometer (RM9-PD, Ophir) (see Section 5 in Supporting Information). The light transmitted through the dichroic mirror was used to image the sample plane with a CMOS camera and a camera lens (MVL50M23) for sample alignment.

In order to gain a complete picture of the spectral SHG responses of the samples, we performed nonlinear experiments while scanning the wavelength of the fundamental (SHG) beam from 1000 to $1300 \mathrm{~nm}$ (from 500 to $650 \mathrm{~nm}$ ) (see Figure $4 a)$. The symmetry of the samples $\left(C_{1 v}\right)$ dictates that

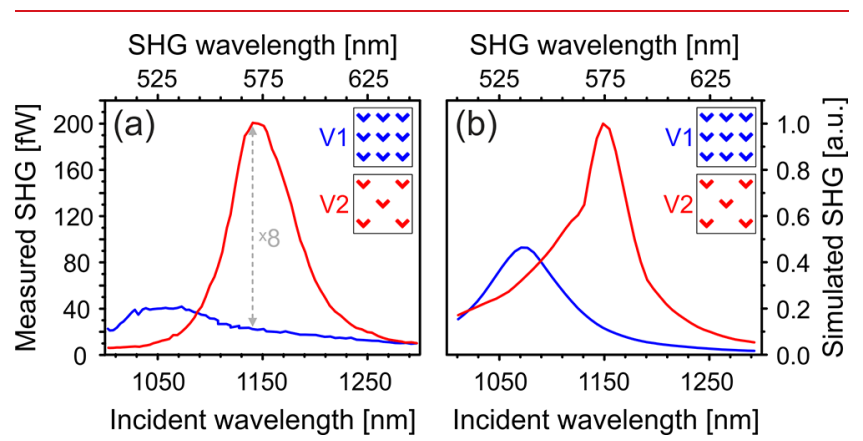

Figure 4. Measured (a) and simulated (b) wavelength-dependent emitted SHG signals for the metasurfaces V1 (blue) and V2 (red). Gray dashed double-side arrow shows the difference in the SHG at $1135 \mathrm{~nm}$, where the optical densities of the arrays are equal. The simulations are performed with the DDA method.

the nonvanishing second-order susceptibility tensor components are $\left(\chi_{y y y}^{(2)}, \chi_{y x x}^{(2)}, \chi_{x y x}^{(2)}=\chi_{x x y}^{(2)}\right)$. To simplify the analysis of our results, the following discussion is focused solely on the tensor component $\chi_{y y y}^{(2)}$, which is both allowed by symmetry and exhibits a resonance that we were able to experimentally access using the OPO (shaded red area in Figure 2). At first glance, the SHG responses follow the intuitively expected behavior where the highest SHG efficiency occurs approximately at the wavelengths of the $y$-polarized resonances $(1081 \mathrm{~nm}$ for V1 and $1151 \mathrm{~nm}$ for V2; compare Figures 2a and 4a). The slight blueshift of the SHG maximum with respect to the maximum of the LSPRs agrees with previous observations. ${ }^{35}$ Larger discrepancy from the expected behavior is observed in the case of sample V1, because the spectral SHG responses are broader and less intense than expected by looking at the respective linear response (see Figure 2a). A simple qualitative comparison of SHG responses shows clearly that the sample V2 exhibits a considerably stronger SHG response than the sample V1. More specifically, the response of V2 is enhanced by a factor of 5.4 compared to the sample V1 at the respective maxima of the two samples. In addition, at the wavelength where both samples have equal optical densities $(1135 \mathrm{~nm})$, V2 demonstrates over 8 -fold increase in the detected SHG signal (see gray dashed double-side arrow in Figure 4a).

The resonant spectral features of metasurfaces are well described by the Lorentz model. ${ }^{35,46}$ Indeed, as a first approximation, close to the plasmon resonance at the 
Table 1. Parameters of the Resonances and SHG Responses [at Linecenters $(1081 \mathrm{~nm}, \mathrm{V1} ; 1151 \mathrm{~nm}, \mathrm{V2})$ and at $1135 \mathrm{~nm}$ ] of Samples V1 and $\mathrm{V}^{a}$

\begin{tabular}{|c|c|c|c|c|c|c|c|c|}
\hline \multirow[b]{2}{*}{ sample } & \multirow[b]{2}{*}{$\begin{array}{l}y \text {-polarized resonance } \\
\text { (linecenter) }[\mathrm{nm}]\end{array}$} & \multirow[b]{2}{*}{$\begin{array}{l}\text { fwhm } \\
{[\mathrm{nm}]}\end{array}$} & \multirow[b]{2}{*}{$\begin{array}{l}\text { line width of the } \\
\text { resonance }\end{array}$} & \multirow[b]{2}{*}{$\begin{array}{l}\text { number } \\
\text { density } N\end{array}$} & \multicolumn{2}{|c|}{$\begin{array}{l}\text { normalized SHG scaling } \\
\text { factor }\end{array}$} & \multicolumn{2}{|c|}{$\begin{array}{l}\text { normalized SHG } \\
\text { intensity }(y y y)\end{array}$} \\
\hline & & & & & $\begin{array}{c}\text { at } \\
\text { linecenter }\end{array}$ & $\begin{array}{c}\text { at } \\
1135 \mathrm{~nm}\end{array}$ & $\begin{array}{c}\text { at } \\
\text { linecenter }\end{array}$ & $1135 \mathrm{~nm}$ \\
\hline V1 & 1081 & 108 & $\gamma$ & 1 & 1 & 1 & 1 & 1 \\
\hline V2 & 1151 & 76 & $0.7 \gamma$ & 0.5 & 1.02 & 2.94 & 5.01 & 8.54 \\
\hline
\end{tabular}

${ }^{a}$ The scaling factors are calculated using Lorentz model (eq 1) and the SHG intensities are obtained from the measurements (Figure 4). The numbers are normalized to the respective ones for sample V1.

fundamental wavelength, the $\mathrm{SH}$ responses of metasurfaces can be described by the relation

$$
\chi_{\text {eff }}^{(2)} \sim \frac{N}{(\Delta-i \gamma)^{2}}
$$

where $N$ describes the number density of the nanoparticles, $\Delta$ is the detuning of the plasmon linecenter from the fundamental frequency, and $\gamma$ is the line width of the resonance. Consequently, the detected SHG signal intensity is proportional to the square of eq 1 . In this framework, the secondorder susceptibility $\chi_{\text {eff }}^{(2)}$ on resonance $(\Delta=0)$ is proportional to $N$ and inversely proportional to the square of $\gamma$. Therefore, the resonant SHG response can be enhanced either by increasing the number density or by decreasing the line width of the resonance.

We first consider the results at the respective linecenters of the two samples (Table 1). Compared to the reference sample V1 with number density $N$ and line width $\gamma$, the modified sample V2 has number density $N / 2$ and line width $0.7 \gamma$. In consequence, the SHG signals from the two samples should be approximately equal (see Table 1). However, the secondharmonic intensities recorded for the two samples differ by a factor of 5. This already suggests that the Lorentz model alone is not sufficient to explain this observation. Instead the local fields at the particles are also significantly affected by the modified interparticle interactions mediated by the SLRs.

The spectral positions of resonances for both samples are different, therefore we performed similar analysis at the wavelength where both samples have equal optical densities $(1135 \mathrm{~nm})$. At this wavelength the analysis based on the Lorentz model (see eq 1) must include the detuning of the resonances $(\Delta=54 \mathrm{~nm}$ and $\Delta=16 \mathrm{~nm}$ for sample $\mathrm{V} 1$ and V2, respectively). In consequence, due to the smaller detuning $(0.3 \Delta$, comparing to sample V1) the SHG from the sample V2 should be enhanced by a factor of 3 (see Table 1 ). Instead, the SHG signal from sample V2 is stronger by a factor of 8.5. Such enhancement in the SHG response provides further support to the result that the lattice interactions, which are not accounted for in the Lorentz model, play a crucial role in the overall SHG responses and are responsible of the measured enhancement. It is important to underline, that the position of the RA at 1025 $\mathrm{nm}$ (Figure 2a) results from the specific parameters of the sample V2 (period $=707 \mathrm{~nm}$ and refractive index of the substrate 1.45) favoring formation of a SLR, whereas no SLRs are formed in the case of sample V1 (period $=500 \mathrm{~nm})$.

In order to verify the origin of these results, we performed simulations using an approach based on nonlinear discretedipole approximation (DDA), ${ }^{39}$ which takes fully into account the lattice interactions, occurring both at the fundamental and the SHG wavelengths. In this approach, each particle in the array is treated as an electric dipole associated with a polarizability that is extracted from experimental data (Section 2 in Supporting Information). The simulated SHG signal (Figure $4 b$ ) shows very good agreement with the experimental results (Figure 4a). The slight discrepancy arises from the fact that the nanoparticles of finite size are reduced to point dipoles. The excellent agreement between the experimental data and the nonlinear DDA simulations were further confirmed by performing simulations using other numerical tools, namely the finite element method (by COMSOL Multiphysics) and surface integral equation (SIE) method (Sections 3 and 4 in Supporting Information). These additional simulations were performed to provide further numerical support to the relatively novel DDA approach. All simulations show an enhancement of SHG when the lattice interaction is effective and the LSPR line width is reduced. This modification of the LSPR line width results in a 3 -fold enhancement of the fundamental intensity close to the nanoparticles, see Figure S6. Because the fundamental nearfield enhancement around each nanoparticle is stronger, the $\mathrm{SH}$ dipole induced in each nanoparticle is also stronger resulting in enhanced $\mathrm{SH}$ emission for sample V2 despite reduced nanoparticle density. In other words, a proper design of the lattice interactions and fundamental field enhancement is able to overcome a lower $\mathrm{SH}$ emitter density.

Finally, we estimate the conversion efficiency of the sample V2 to be around $2.5 \times 10^{-11}$ for the input beam power of 8 $\mathrm{mW}$. Although the value is seemingly small when compared to conventional nonlinear crystals, it is highly promising for such a thin metasurface $(\sim 20 \mathrm{~nm})$ to be excited using moderate peak intensities. ${ }^{29}$ If higher peak intensities could be utilized, considerably higher conversion efficiencies would be achieved. $^{47,48}$ Ultimately, the conversion efficiency is mostly restricted by the material damage via heating. Interestingly, recent work shows that peak intensities even up to $10 \mathrm{GW} / \mathrm{cm}^{2}$ should be possible by using properly coated plasmonic structures. ${ }^{49}$ Therefore, we are confident that considerable improvements could be achieved by carefully designing structures that could sustain stronger pump powers.

In conclusion, we have shown that second-harmonic generation from metasurfaces can be significantly enhanced by proper designs of the lattice interactions leading to strong light-matter interactions in the arrays through enhanced local fields. We have verified this principle by investigating two metasurfaces consisting of identical V-shaped nanoparticles, which have different particle number density and arrangement. The resonant second-order nonlinear response of the modified sample was over five times stronger than that of the reference sample. Surprisingly, such a considerable enhancement was achieved while halving the particle number density of the sample array. We believe that these results open new 
possibilities for the fabrication of more efficient nonlinear metamaterials.

\section{ASSOCIATED CONTENT}

\section{S Supporting Information}

The Supporting Information is available free of charge on the ACS Publications website at DOI: 10.1021/acs.nanolett.8b03378.

Extinction spectra and near fields calculated using FEM; nonlinear DDA simulations of all four sample arrays; SIE method modeling for studied samples; FEM modeling of SHG; power calibration of used detector; power dependence of the SHG and estimates of the damage threshold level; verification of the SHG experiment (PDF)

\section{AUTHOR INFORMATION}

\section{Corresponding Authors}

*E-mail: robert.czaplicki@fizyka.umk.pl.

*E-mail: martti.kauranen@tut.fi.

\section{ORCID $\odot$}

Robert Czaplicki: 0000-0003-1197-1614

Jérémy Butet: 0000-0001-9598-9074

Olivier J. F. Martin: 0000-0002-9574-3119

\section{Author Contributions}

The manuscript was written through contributions of all authors. All authors have given approval to the final version of the manuscript.

\section{Notes}

The authors declare no competing financial interest.

\section{ACKNOWLEDGMENTS}

We gratefully acknowledge financial support from Academy of Finland (265682, 287651, 308596).

\section{REFERENCES}

(1) Maier, S. A. Plasmonics: fundamentals and applications; Springer: New York, 2007.

(2) Boardman, A. D.; Grimalsky, V. V.; Kivshar, Y. S.; Koshevaya, S. V.; Lapine, M.; Litchinitser, N. M.; Malnev, V. N.; Noginov, M.; Rapoport, Y. G.; Shalaev, V. M. Laser Photon. Rev. 2011, 5, 287-307.

(3) Kauranen, M.; Zayats, A. V. Nat. Photonics 2012, 6, 737-748.

(4) Meinzer, N.; Barnes, W. L.; Hooper, I. R. Nat. Photonics 2014, 8, 889-898.

(5) Koenderink, A. F.; Alù, A.; Polman, A. Science 2015, 348, 516521.

(6) Butet, J.; Brevet, P.-F.; Martin, O. J. F. ACS Nano 2015, 9, $10545-10562$.

(7) Metzger, B.; Hentschel, M.; Giessen, H. ACS Photonics 2016, 3, $1336-1350$

(8) Chen, H.-T.; Taylor, A. T.; Yu, N. Rep. Prog. Phys. 2016, 79, No. 076401.

(9) Li, G.; Zhang, S.; Zentgraf, T. Nat. Rev. Mater. 2017, 2, 17010.

(10) Linden, S.; Niesler, F. B. P.; Förstner, J.; Grynko, Y.; Meier, T.; Wegener, M. Phys. Rev. Lett. 2012, 109, No. 015502.

(11) Chu, Y.; Schonbrun, E.; Yang, T.; Crozier, K. B. Appl. Phys. Lett. 2008, 93, 181108.

(12) Auguié, B.; Barnes, W. L. Phys. Rev. Lett. 2008, 101, 143902.

(13) Kravets, V. G.; Schedin, F.; Grigorenko, A. N. Phys. Rev. Lett. 2008, 101, No. 087403.

(14) Vecchi, G.; Giannini, V.; Gómez Rivas, J. Phys. Rev. B: Condens. Matter Mater. Phys. 2009, 80, 201401.
(15) Murai, S.; Verschuuren, M. A.; Lozano, G.; Pirruccio, G.; Rodriguez, S. R. K.; Gómez Rivas, J. Opt. Express 2013, 21, 42504262.

(16) Swiecicki, S. D.; Sipe, J. E. Phys. Rev. B: Condens. Matter Mater. Phys. 2017, 95, 195406.

(17) Kwiat, P. G.; Mattle, K.; Weinfurter, H.; Zeilinger, A.; Sergienko, A. V.; Shih, Y. Phys. Rev. Lett. 1995, 75, 4337-4341.

(18) Kippenberg, T. J.; Holzwarth, R.; Diddams, S. A. Science 2011, $332,555-559$.

(19) Czaplicki, R.; Mäkitalo, J.; Siikanen, R.; Husu, H.; Lehtolahti, J.; Kuittinen, M.; Kauranen, M. Nano Lett. 2015, 15, 530-534.

(20) Bouhelier, A.; Beversluis, M.; Hartschuh, A.; Novotny, L. Phys. Rev. Lett. 2003, 90, No. 013903.

(21) Nappa, J.; Revillod, G.; Russier-Antoine, I.; Benichou, E.; Jonin, C.; Brevet, P.-F. Phys. Rev. B: Condens. Matter Mater. Phys. 2005, 71, 165407.

(22) Nappa, J.; Russier-Antoine, I.; Benichou, E.; Jonin, C.; Brevet, P.-F. J. Chem. Phys. 2006, 125, 184712.

(23) Butet, J.; Duboisset, J.; Bachelier, G.; Russier-Antoine, I.; Benichou, E.; Jonin, C.; Brevet, P.-F. Nano Lett. 2010, 10, 17171721.

(24) Butet, J.; Bachelier, G.; Russier-Antoine, I.; Jonin, C.; Benichou, E.; Brevet, P.-F. Phys. Rev. Lett. 2010, 105, No. 077401.

(25) Zhang, Y.; Grady, N. K.; Ayala-Orozco, C.; Halas, N. J. Nano Lett. 2011, 11, 5519-5523.

(26) Canfield, B.; Kujala, K.; Jefimovs, K.; Turunen, J.; Kauranen, M. Opt. Express 2004, 12, 5418-5423.

(27) Klein, M. W.; Enkrich, C.; Wegener, M.; Linden, S. Science 2006, 313, 502-504.

(28) Canfield, B. K.; Kujala, S.; Jefimovs, K.; Svirko, Y.; Turunen, J.; Kauranen, M. J. Opt. A: Pure Appl. Opt. 2006, 8, S278-S284.

(29) (a) Klein, M. W.; Wegener, M.; Feth, N.; Linden, S. Opt. Express 2007, 15, 5238-5247. (b) Klein, M. W.; Wegener, M.; Feth, N.; Linden, S. Opt. Express 2008, 16, 8055-8055.

(30) Kujala, S.; Canfield, B. K.; Kauranen, M.; Svirko, Y.; Turunen, T. Phys. Rev. Lett. 2007, 98, 167403.

(31) Valev, V. K.; Silhanek, A. V.; Verellen, N.; Gillijns, W.; Van Dorpe, P.; Aktsipetrov, O. A.; Vandenbosch, G. A. E.; Moshchalkov, V. V.; Verbiest, T. Phys. Rev. Lett. 2010, 104, 127401.

(32) Niesler, F. B. P.; Feth, N.; Linden, S.; Wegener, M. Opt. Lett. 2011, 36, 1533-1535.

(33) Gentile, M.; Hentschel, M.; Taubert, R.; Guo, H.; Giessen, H.; Fiebig, M. Appl. Phys. B: Lasers Opt. 2011, 105, 149-162.

(34) Butet, J.; Gallinet, B.; Thyagarajan, K.; Martin, O. J. F. J. Opt. Soc. Am. B 2013, 30, 2970-2979.

(35) Metzger, B.; Gui, L.; Fuchs, J.; Floess, D.; Hentschel, M.; Giessen, H. Nano Lett. 2015, 15, 3917-3922.

(36) Czaplicki, R.; Zdanowicz, Z.; Koskinen, K.; Laukkanen, J.; Kuittinen, M.; Kauranen, M. Opt. Express 2011, 19, 26866-26871.

(37) Sönnichsen, C.; Franzl, T.; Wilk, T.; von Plessen, G.; Feldmann, J.; Wilson, O.; Mulvaney, P. Phys. Rev. Lett. 2002, 88, No. 077402.

(38) Zou, S.; Janel, N.; Schatz, G. C. J. Chem. Phys. 2004, 120, $10871-10875$.

(39) Huttunen, M. J.; Dolgaleva, K.; Törmä, P.; Boyd, R. W. Opt. Express 2016, 24, 28279-28289.

(40) Czaplicki, R.; Kiviniemi, A.; Laukkanen, J.; Lehtolahti, J.; Kuittinen, M.; Kauranen, M. Opt. Lett. 2016, 41, 2684-2687.

(41) Michaeli, L.; Keren-Zur, S.; Avayu, O.; Suchowski, H.; Ellenbogen, T. Phys. Rev. Lett. 2017, 118, 243904.

(42) Huttunen, M. J.; Rasekh, P.; Boyd, R. W.; Dolgaleva, K. Phys. Rev. A: At., Mol., Opt. Phys. 2018, 97, No. 053817.

(43) Mäkitalo, J.; Suuriniemi, S.; Kauranen, M. Opt. Express 2011, 19, 23386-23399.

(44) Jin, J. M. The Finite Element Method in Electromagnetics; WileyIEEE, 2014

(45) Husu, H.; Mäkitalo, J.; Laukkanen, J.; Kuittinen, M.; Kauranen, M. Opt. Express 2010, 18, 16601-16606. 
(46) Boyd, R. W. Nonlinear Optics, 3rd ed.; Academic Press: San Diego, 2008.

(47) Nezami, M. S.; Gordon, R. Opt. Express 2015, 23, 3200632014.

(48) Nezami, M. S.; Yoo, D.; Hajisalem, G.; Oh, S.-H.; Gordon, R. ACS Photonics 2016, 3, 1461-1467.

(49) Albrecht, G.; Kaiser, S.; Giessen, H.; Hentschel, M. Nano Lett. 2017, 17, 6402-6408. 